\title{
Dagli sciamani allo sciamanesimo. Discorsi, credenze, pratiche
}

("Quality Paperbacks", 517), Roma, Carocci, 2018, pp. 172, ISBN 9788843090839, € 15,00

\section{Giovanni Ingarao}

\section{(2) OpenEdition}

\section{Journals}

\section{Edizione digitale}

URL: http://journals.openedition.org/mythos/362

DOI: $10.4000 /$ mythos.362

ISSN: 2037-7746

\section{Editore}

Salvatore Sciascia Editore

\section{Edizione cartacea}

Data di pubblicazione: 1 dicembre 2018

Paginazione: 148-149

ISBN: 978-88-8241-501-3

ISSN: $1972-2516$

\section{Notizia bibliografica digitale}

Giovanni Ingarao, «Dagli sciamani allo sciamanesimo. Discorsi, credenze, pratiche », Mythos [Online], 12 | 2018, online dal 24 septembre 2019, consultato il 23 septembre 2020. URL : http:// journals.openedition.org/mythos/362 ; DOI : https://doi.org/10.4000/mythos.362 


\section{Sergio Botta}

Dagli sciamani allo sciamanesimo. Discorsi, credenze, pratiche

(“Quality Paperbacks", 517), Roma, Carocci, 2018, pp. 172, ISBN 9788843090839, €15,00.

\section{Giovanni Ingarao - Università degli Studi di Palermo - gioinga@hotmail.it}

L'ultimo libro di S. Botta è un'ottima guida per chiunque volesse confrontarsi con un argomento complesso che ha interessato il panorama accademico ottocentesco e novecentesco e che tutt'oggi tocca diverse realtà della società contemporanea. Come illustra bene l'autore, con uno stile chiaro ed efficace che accompagna il lettore per centocinquanta pagine, lo sciamanesimo è stato progressivamente discostato dal suo luogo d'origine per divenire un fenomeno estremamente fluido, declinabile in modi, luoghi e tempi estremamente differenti da loro. Quest'operazione costringe a porsi alcune fondamentali questioni metodologiche che dovrebbero concernere tutti gli studiosi umanistici. Fino a che punto è lecito adottare una categoria analitica, che nasce in un preciso contesto storico-culturale, per descrivere fenomeni diversi fra loro? Quale legame è possibile identificare tra manifestazioni piuttosto lontane fra loro nel tempo e nello spazio? Da chi, con quale sguardo e con quali intenzioni vengono tracciati i confini denotativi tra un contesto e l'altro? Domande che inevitabilmente toccano altri temi scottanti, primo tra tutti il neocolonialismo degli occidentali che spesso cercano di "introiettare, comprendere, depotenziare e, infine, addomesticare" ciò che è estraneo (149).

Botta ci dà al contempo la possibilità di compiere un percorso diacronico ricco e lungo che va ben oltre i confini della Siberia. È interessante scoprire che questo fenomeno religioso ha coinvolto, fra gli altri, importanti intellettuali illuministi e romantici, come D. Diderot e J. G. Herder, e ha riguardato discipline piuttosto diverse fra loro: I'antropologia, l'etnografia e la storia delle religioni, ovviamente, ma anche la filosofia, la psicolo- gia, la sociologia, l'arte, la poesia e persino la storia degli antichi Greci. I celebri studi di K. Meuli, Scythica (Hermes 70, 1938, 121176) e di E. R. Dodds, The Greek Shamans and the Origin of Puritanism (The Greeks and the Irrational, Berkeley-Los Angeles 1951, 135-178), hanno suscitato infatti un vivo dibattito anche tra gli antichisti, a proposito dell'opportunità di adottare termini e concetti simili per interpretare il mondo greco antico. Lo sciamanesimo è stato connesso alle origini stesse dell'espressione poetica, intesa come nucleo dell'epica indoeuropea e ugrofinnica e, andando ancora più indietro nel tempo, è stata persino proposta un'interpretazione sciamanica dell'arte parietale preistorica (Sciamani dell'antichità e sciamani della preistoria, 115-118).

Alcune tra le menti più brillanti del Novecento si sono appassionate allo sciamanesimo. Non mi riferisco solo a 'specialisti' come Mircea Eliade che, con la sua famosa e controversa monografia Le chamanisme et les techniques archaïques de l'extase (Paris 1951), ha costituito un fondamentale punto di riferimento nella storia degli studi sul tema. Grandissimi pensatori, quali C. Lévi-Strauss e C. G. Jung, si sono interessati vivamente alla materia, contribuendo attivamente al dibattito che ha coinvolto, sebbene in parte, anche la realtà italiana. Ernesto De Martino e Carlo Ginzburg vanno annoverati, di certo, tra le figure di spicco del panorama culturale nostrano che hanno affrontato questo argomento così discusso. II viaggio che S. Botta propone è anche un itinerario che tocca inevitabilmente realtà geografiche e sociali molto differenti tra loro. Dopo alcune (necessarie) riflessioni più teoriche (cap. 1 Definire lo sciamanesimo), lo studioso ci porta alla scoperta degli sciamani siberiani 
e cerca di indagare anche le manifestazioni antecedenti alla costruzione di uno sguardo occidentale (cap. 2 La scoperta degli sciamani). Passando attraverso il contrastato rapporto degli zar russi con lo sciamanesimo (cap. 3 L'invenzione dello sciamanesimo), si arriva al decisivo 'sbarco' di questo fenomeno religioso nelle Americhe grazie al fondamentale contributo di Franz Boas (cap. 4 La diffusione nelle Americhe). L'etnografo tedesco, molto attivo negli Stati Uniti, fu l'artefice di un'importante spedizione esplorativa, la Jesup North Pacific Expedition (18971902), composta da specialisti americani e russi, che avevano l'obiettivo di comprendere se ci fossero dei legami tra le culture della Siberia nord-orientale e quelle della costa nord-occidentale del continente americano. Boas voleva dimostrare l'esistenza di un "complesso sciamanico" condiviso dalle culture indigene di entrambi i paesi (73). In tal modo fu avviata un'operazione di generalizzazione che allargò la prospettiva a realtà altre rispetto alla Siberia.

Le interpretazioni psicopatologiche (cap. 5 Psicopatologia, medicalizzazione, de-medicalizzazione, cura) non hanno di certo fermato il successo dello sciamanesimo che, come accennavo, ha interessato fra le altre discipline anche la psicanalisi (cap. 6 Arcaicizzazione e idealizzazione). La sua grande diffusione nella controcultura statunitense (cap. 7 II neosciamanesimo come individualizzazione e istituzionalizzazione) è divenuta infine il trampolino di lancio di un "eroe culturale eclettico che parla, ancora oggi, a quegli individui solitari alla ricerca delle tecniche per intraprendere un'avventura psichica che consenta loro di diffondere la propria sensibilità nell'ambiente" (120). II successo mondiale dei libri di Carlos Castaneda, che furono duramente contestati da gran parte del mondo accademico, trasformò lo sciamanesimo in "merce globale", grazie anche alla diffusione di un metodo pratico a scopo di lucro (130). Anche la cultura psichedelica ne fu attratta, in connessione all'uso di sostanze psicotrope che ha portato addirittura a una sorta di turismo "mistico sciamanico" nei territori indigeni (125). In tal modo si è lentamente arrivati a una mercificazione della dimensione religiosa, in risposta alla quale i gruppi indigeni stanno oggi riappropriandosi delle loro credenze e della loro identità (cap. 8 II ritorno nei contesti indigeni), grazie proprio allo sciamanesimo che "è divenuto uno strumento - globalmente riconosciuto - attraverso il quale rivendicare gli interessi condivisi di una moltitudine di popolazioni autoctone" (149).

Come spiega l'autore, nelle conclusioni, lo sciamanesimo è diventato una categoria capace di attrarre desideri e bisogni, individuali e collettivi, molto diversi tra loro (149). Dagli sciamani allo sciamanesimo ci fornisce un ottimo sguardo d'insieme su un argomento ostico, senza mai dimenticare che le operazioni interpretative trattate nel volume sono frutto della proiezione dello sguardo occidentale su fenomeni locali che hanno poi assunto una dimensione molto più vasta. Botta ci mette così a disposizione un utilissimo strumento di lavoro che risponde pienamente agli obiettivi della collana Quality Paperbacks in cui il testo è stato pubblicato: un libro tascabile di qualità, non solo per gli specialisti, ma per chiunque volesse allargare il proprio orizzonte conoscitivo. 